\title{
Infrared gluons in the stochastic quantization approach
}

\section{Yoshiyuki Nakagawa}

Research Center for Nuclear Physics, Osaka University, Ibaraki, Osaka 567-0047, Japan

\section{Atsushi Nakamura}

Research Institute for Information Science and Education, Hiroshima University,

Higashi-Hiroshima 739-8521, Japan

\section{Takuya Saito*}

Integrated Information Center, Kochi University, Kochi, 780-8520, Japan

E-mail: tsaitou@kochi-u.ac.jp

Hiroshi Toki

Research Center for Nuclear Physics, Osaka University, Ibaraki, Osaka 567-0047, Japan

\begin{abstract}
We study the infrared behavior of the transverse gluon propagators by using the lattice $S U(3)$ calculation in the stochastic quantization approach with the Coulomb gauge. As the lattice volume increases, the infrared gluon propagator shows sign of suppression near $p=0$. However, the infrared gluon is strongly affected with variation of a gauge parameter.
\end{abstract}

The XXVI International Symposium on Lattice Field Theory

July 14-19 2008

Williamsburg, Virginia, USA

*Speaker. 


\section{Introduction}

To understand the confinement phenomena of quantum chromodynamics (QCD) is one of the most challenging problems in modern physics. The gluons and quarks are confined in hadrons where a non-perturbative mode dominates. In such confinement regions it is expected that the transverse mode of gluons will be suppressed. The Landau-gauge gluon propagator calculated by the large-lattice computation ( $S U(2)[1]$ and $S U(3)$ [2] ) seems to have a flat point in the infrared limit, not to be suppressed. In addition, in the case of the Coulomb gauge, the similar result has been obtained in Ref. [3, 4], while more significant suppression has been observed in Ref. [5].

In this study a stochastic quantization with the Coulomb-gauge fixing term $[6,7]$ is employed in order to obtain an observation on the infrared suppression of the transverse gluons left as a problem. This method has some advantages in comparison with path-integral formulation: 1) This does not require an iterative technique for fixing a gauge, so that one can expect the computational time in advance; particularly, it may be desirable for large-lattice computations. 2) The gaugefixing term introduced by Zwanziger makes all the gauge configurations automatically go to the Gribov region; that is, this term works as an attractive force. 3) The gauge-parameter $\alpha$ can be changed at will, investigating the dependence on $\alpha$ for measurements. This algorithm has been already applied to the gluon screening-mass calculation in Ref. [8]

A confinement dynamics of Coulomb gauge QCD has been discussed in the framework of a Gribov-Zwanziger scenario $[9,10]$, which has been looked into recently. Although the infrared suppression of the Coulomb-gauge transverse gluons has not been confirmed in the lattice simulation, there are a lot of progress for it. In particular, the $S U(2)$ and $S U(3)$ calculations show that a colorCoulomb (instantaneous ) interaction provides a linearly rising potential at large distances, whose string tensions are $2-3$ times larger than the usual Wilson-loop string tension[11, 12, 13, 14]. This results are expected from an inequality, $V_{p}(R)<V_{c}(R)$, where $V_{p}(R)$ and $V_{c}(R)$ are a physical potential and the color-Coulomb potential [16]. This inequality is a necessary condition for the color confinement; moreover, this remains even in the deconfinement phase [12, 13, 14]. In addition, it is confirmed that the effective string tension estimated by the color-Coulomb propagators is also in agreement with this inequality[15]. Furthermore, in the Gribov-Zwanziger scenario, the strong singularity of the confining gluons results from an infrared enhancement of Faddeev-Popov (FP) operator of QCD. The practical numerical simulations $[17,18]$ show that the density of the low-lying eigenvalues increases with the lattice volume and the confinement criterion is satisfied. Meanwhile, the color-Coulomb string tension is trying to be reproduced by a spectral-sum method for the FP propagator [19].

\section{Stochastic gauge fixing}

The stochastic quantization is based on the Langevin equation that introduces virtual time in addition to the Euclidean coordinate. Zwanziger introduced a gauge-fixing term as

$$
\frac{d A_{\mu}^{a}}{d \tau}=-\frac{\delta S}{\delta A_{\mu}^{a}}+\frac{1}{\alpha} D_{\mu}^{a b}(A) \partial_{v} A_{v}^{b}+\eta_{\mu}^{a}
$$


where $D_{\mu}^{a b}(A)$ is the covariant derivative, $\tau$ is the Langevin time, and $\eta$ is the Gaussian noise term. The second term on the r.h.s. is a gauge-fixing term and the $\alpha$ is a gauge-fixing term. Here the Coulomb-gauge condition $\partial_{i} A_{i}^{a}=0$ is adopted.

Additionally, Mizutani and Nakamura formulated this equation into a lattice version [20]. The link variables, $U_{\mu}$, are rotated through the following gauge transformation depending on $\tau$,

$$
U_{\mu}(x, \tau+\Delta \tau)=\omega^{\dagger}(x, \tau) e^{i f_{\mu}^{a} t^{a}} U_{\mu}(x, \tau) \omega(x+\hat{\mu}, \tau) .
$$

Here, $f_{\mu}^{a}$ stand for a driving force,

$$
f_{\mu}^{a}=-\frac{\partial S}{\partial A_{\mu}^{a}} \Delta \tau+\eta^{a} \sqrt{\Delta \tau}
$$

and the gauge rotation is given by

$$
\omega=e^{i \beta \Delta^{a} \tau^{a} \Delta \tau / \alpha} .
$$

If $\omega=I$, Eq. (2.2) is a Langevin process on the lattice. Eq. (2.2) with Eq. (2.4) leads to the gaugefixing term as $\Delta \tau \rightarrow 0$. Eq. (2.2) means that the gauge rotation and Langevin step are executed alternately.

\section{Coulomb gauge QCD}

The Hamiltonian of QCD in the Coulomb gauge can be given by

$$
H=\frac{1}{2} \int d^{3} x\left(E_{i}^{2}(\vec{x})+B_{i}^{2}(\vec{x})\right)+\frac{1}{2} \int d^{3} x d^{3} y(\rho(\vec{x}) \mathscr{V}(\vec{x}, \vec{y}) \rho(\vec{y})),
$$

where $E_{i}, B_{i}$ and $\rho$ are the transverse electric fields, the transverse magnetic fields and the colorcharge density, respectively. The function $\mathscr{V}$ in the second term is made by the FP operator, $M=$ $-\vec{D} \vec{\partial}=-\left(\vec{\partial}^{2}+g \vec{A} \times \vec{\partial}\right)$,

$$
\mathscr{V}(\vec{x}, \vec{y})=\int d^{3} z\left[\frac{1}{M(\vec{x}, \vec{z})}\left(-\vec{\partial}_{(\vec{z})}^{2}\right) \frac{1}{M(\vec{z}, \vec{y})}\right] .
$$

In the Coulomb gauge, the transverse (first) term makes a physical gluon field. In the confinement phase, one can expect that the transverse gluon in the infrared limit vanishes due to the gluon mass going to infinity effectively. On the other hand, the source term gives a color-Coulomb instantaneous (confining) potential, which is required to build a bound state out of quarks. In addition, the confining linearity of this potential results from accumulation of the low-lying eigenvalues of the FP operator. Besides, the QCD in the Coulomb gauge has no negative norm and thus a physical interpretation is very clear. As a result, the $q q$ (diquark) interaction in the color- $3^{*}$ channel was calculated in this framework [21].

\section{Gluon propagators}

In the continuum theory the gluon propagators in the spatial direction are formulated as 


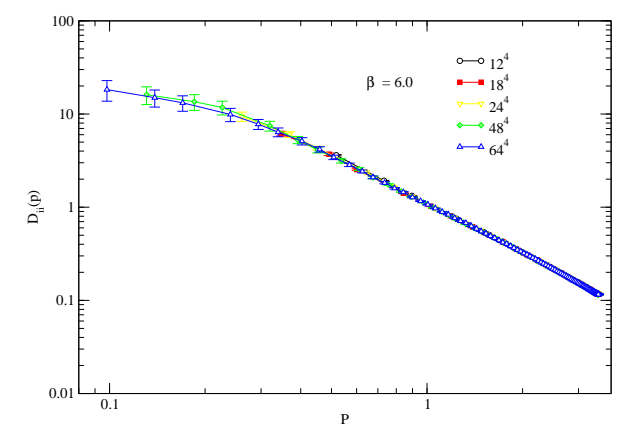

Figure 1: Volume dependence of $D(p)$

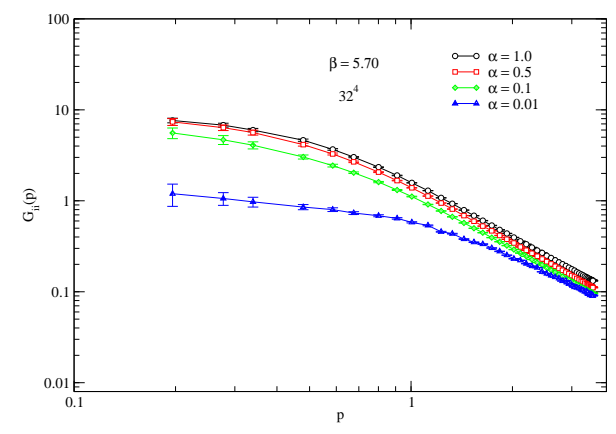

Figure 2: Gauge-parameter $\alpha$ dependence of $D(p)$

$$
D_{i j}^{a b}(\vec{q})=\delta^{a b}\left[\delta_{i j}-\left(\frac{q_{i} q_{j}}{\vec{q}^{2}}\right) D\left(\vec{q}^{2}\right)\right]+\alpha\left(\frac{q_{i} q_{j}}{\vec{q}^{2}}\right) D^{L}\left(\vec{q}^{2}\right) .
$$

We are now interested in the transverse $D(p)$. For free-theory case, we have $D_{0}\left(q^{2}\right)=1 / q^{2}$ which diverges in the infrared limit. Meanwhile, if quarks are confined by the linear potential there are no general formulations for it. Nevertheless, the expectation $D\left(q^{2}=0\right)=0$ has been discussed by many authors (See Ref. [22] ).

On the lattice, the gluon (gauge) fields are defined as

$$
A_{i}^{a}(x)=2 \operatorname{Im} \operatorname{Tr}\left(U_{i}(x) T^{a}\right)
$$

where $T^{a}$ are color-SU(3) matrices. In momentum space, the two-point function is given by

$$
D_{i j}^{a b}(q)=\left\langle A_{i}^{a}(p) A_{j}^{b}(-p)\right\rangle
$$

which are going to be measured here.

\section{Numerical results}

Here the gauge configurations are generated by using the quenched Wilson gauge action in the stochastic quantization with the Coulomb gauge. The gauge coupling $\beta \mathrm{s}$ are 6.0 and 5.7. For the small lattice, $L=12,18,24,32$, after the 10k thermalization done, 100 gauge configurations measured every 100 Langevin steps are used. In contrast, for the large lattice, $L=48,64$, the $4 \mathrm{k}$ thermalization and 40 configurations (10 steps separated ) are carried out. The Langevin step size is fixed as only $\Delta \tau=0.01$; however, the gluon propagator does not strongly depend on $\Delta \tau$ as shown in Ref. [8].

A numerical result of $D_{i i}(p)$ with $\alpha=1$ at $\beta=6.0$ is shown in Fig. 1, in which the largest lattice-volume size is approximately $(6.4 \mathrm{fm})^{4}$ for $64^{4}$. It is found that in the vanishing momentum $D_{i i}(p)$ are not suppressed but do not diverge. 
The gauge-parameter dependence of the gluon propagators is shown in Fig. 2. Here, for $\alpha=1.0,0.5$ and $0.1, \Delta \tau=0.01$, while for $\alpha=0.01, \Delta \tau=0.001$. In the high-momentum regions, the variation with $\alpha$ is very small, and so they are comparable; however, in the infrared region, their behavior changes significantly with $\alpha$. In particular, the smallest- $\alpha$ calculation gives a better result.

\section{Summary}

We have calculated the gluon propagators in the confinement regions in the stochastic Coulombgauge fixing. Our results show that the spatial-gluon propagator becomes flat in the infrared limit. Moreover, we find that the infrared behavior of gluon is very sensitive to variation of gaugeparameter $\alpha$.

In order to improve our results it may be important to know the $\alpha$-parameter dependence more extensively; for example, in addition to the gluon propagators, how the FP propagator (eigenvalue) depends on $\alpha$. Notice that the computational cost seriously increases as $\alpha$ decreases. This is the reason why the gauge-fixing term in our algorithm is proportional to a factor $\sim \Delta \tau / \alpha$, which has to be small enough to carry out numerical steps accurately.

\section{References}

[1] A. Cucchieri, T. Mendes, PoS (LATTICE 2007) 297.

[2] I.L. Bogolubsky, E.-M.IIgenfritz, M. Müller-Preussker, A. Sternbeck, PoS (LATTICE 2007) 290.

[3] A. Cucchieri, D. Zwanziger, Phys. Rev. D 65, 014001 (2001).

[4] Y. Nakagawa, A. Nakamura, T. Saito, H. Toki, PoS (LATTICE 2007) 319.

[5] M. Quandt, G. Burgio, S. Chimchinda, H. Reinhardt, PoS (LATTICE 2007) 325.

[6] D. Zwanziger, Nucl. Phys. B192, 259 (1981).

[7] E. Seiler, I.O. Stamatescu and D. Zwanziger, Nucl. Phys. B239, 177 (1984).

[8] A. Nakamura, T. Saito, S. Sakai, Phys.Rev. D69 (2004) 014506; A. Nakamura, I. Pushkina, T. Saito, S. Sakai, Phys.Lett. B549 (2002) 133.

[9] V. N. Gribov, Nucl. Phys. B139, 1 (1978).

[10] D. Zwanziger, Nucl. Phys. B 518 (1998) 237-272.

[11] J. Greensite and Š. Olejník, Phys. Rev. D56, 094503 (2003), arXiv:hep-lat/0209068.

[12] J. Greensite, Š. Olejník and D. Zwanziger, Phys. Rev. D69, 074506(2004), arXiv:hep-lat/0401003.

[13] A. Nakamura, T. Saito, Prog.Theor.Phys. 115 (2006) 189-200.

[14] Y. Nakagawa, A. Nakamura, T. Saito, H. Toki, D. Zwanziger, Phys.Rev. D73 (2006) 094504.

[15] A. Voigt, E.-M. Ilgenfritz, M. Müller-Preussker, A. Sternbeck, Phys.Rev. D78, 014501 (2008).

[16] D. Zwanziger, Phys. Rev. Lett. 90 (2003) 102001, arXiv:hep-lat/0209105.

[17] J. Greensite, Š. Olejník and D. Zwanziger, JHEP 0505 (2005) 070.

[18] Y. Nakagawa, A. Nakamura, T. Saito, H. Toki, Phys. Rev. D75 (2007) 014508. 
[19] Y. Nakagawa, A. Nakamura, T. Saito, H. Toki, in progress.

[20] A. Nakamura and M. Mizutani, Vistas in Astronomy (Pergamon Press), vol. 37, 305 (1993); M. Mizutani and A. Nakamura, Nucl. Phys. B(Proc. Suppl.) 34, 253 (1994).

[21] Y. Nakagawa, A. Nakamura, T. Saito, H. Toki, Phys.Rev.D77,034015,2008; A. Nakamura, T. Saito, Phys.Lett. B621 (2005) 171.

[22] D. Zwanziger, Nucl. Phys. B364, 1991, 127.

[23] Y. Nakagawa, A. Nakamura, T. Saito, H. Toki, PoS (LATTICE2007) 319. 